\title{
Guidance, Navigation, and Control Performance for the GOES-R Spacecraft
}

\author{
Jim Chapel $^{1}$, Devin Stancliffe ${ }^{1}$, Tim Bevacqua ${ }^{1}$, Stephen Winkler ${ }^{1}$, Brian Clapp
}

Tim Rood ${ }^{2}$, David Gaylor ${ }^{3}$, Doug Freesland ${ }^{4}$, Alexander Krimchansky ${ }^{5}$

${ }^{1}$ Lockheed Martin Space Systems, USA

${ }^{2}$ Advanced Solutions Inc., USA

${ }^{3}$ Emergent Space Technologies, USA

${ }^{4}$ ACS Engineering, USA

${ }^{5}$ NASA/Goddard Space Flight Center, USA

\begin{abstract}
The Geostationary Operational Environmental Satellite-R Series (GOES-R) is the first of the next generation geostationary weather satellites, scheduled for delivery in late 2015 and launch in early 2016. Relative to the current generation of GOES satellites, GOES-R represents a dramatic increase in Earth and solar weather observation capabilities, with 4 times the resolution, 5 times the observation rate, and 3 times the number of spectral bands for Earth observations. GOES-R will also provide unprecedented availability, with less than 120 minutes per year of lost observation time. The Guidance Navigation \& Control (GN\&C) design requirements to achieve these expanded capabilities are extremely demanding. This paper first presents the pointing control, pointing stability, attitude knowledge, and orbit knowledge requirements necessary to realize the ambitious Image Navigation and Registration (INR) objectives of GOES-R. Because the GOES-R suite of instruments is sensitive to disturbances over a broad spectral range, a high fidelity simulation of the vehicle has been created with modal content over $500 \mathrm{~Hz}$ to assess the pointing stability requirements. Simulation results are presented showing acceleration, shock response spectrum (SRS), and line of sight responses for various disturbances from $0 \mathrm{~Hz}$ to $512 \mathrm{~Hz}$. These disturbances include gimbal motion, reaction wheel disturbances, thruster firings for station keeping and momentum management, and internal instrument disturbances. Simulation results demonstrate excellent performance relative to the pointing and pointing stability requirements, with line of sight jitter of the isolated instrument platform of approximately 1 micro-rad. Low frequency motion of the isolated instrument platform is internally compensated within the primary instrument. Attitude knowledge and rate are provided directly to the instrument with an accuracy defined by the Integrated Rate Error (IRE) requirements. The allowable IRE ranges from 1 to 18.5 micro-rad, depending upon the time window of interest. The final piece of the INR performance is orbit knowledge. Extremely accurate orbital position is achieved by GPS navigation at Geosynchronous Earth Orbit (GEO). Performance results are shown demonstrating compliance with the 50 to $75 \mathrm{~m}$ orbit position accuracy requirements of GOES-R, including during station-keeping and momentum management maneuvers. As shown in this paper, the GN\&C performance for the GOES-R series of spacecraft supports the challenging mission objectives of the next generation GEO Earth-observation satellites.
\end{abstract}

\section{GOES-R MISSION OBJECTIVES}

The Geostationary Operational Environmental Satellite-R (GOES-R) is the first of a new generation U.S. geostationary weather satellites, and is scheduled for delivery in late 2015 and launch shortly afterwards. The program is managed collaboratively by the National Oceanic and Atmospheric Administration (NOAA) and the National Aeronautics and Space Administration (NASA). The GOES spacecraft have provided continuous Earth imagery and space weather data for nearly 40 years. The GOES-R series of spacecraft will provide continuity of service as delivered by the current generation of GOES satellites. As such, the mission includes hosting both Earth-observing and space weather instruments, as well as hosting a communication payload to deliver instrument data to the data processing centers, to serve as a data collection platform, and to provide search and rescue services.

Relative to the current generation of GOES satellites, the GOES-R Advanced Baseline Imager (ABI), represents a dramatic increase in Earth weather observation capabilities. ABI will provide 4 times the resolution, 5 times the observation rate, 100 times data rate and 3 times the number of spectral bands for Earth observations [1]. In addition to ABI, a brand new Earth-observing instrument will also be hosted on the spacecraft — Geostationary Lightning Mapper (GLM). GLM is a near-infrared optical transient detector, which can detect extremely brief changes in an optical scene, indicating the presence of lightning. Like ABI, GLM resides on the satellite's Earth Pointed Platform (EPP). The configuration of these instruments on the GOES-R spacecraft is shown in Fig. 1.

Both Earth-pointed instruments demand high performance from the spacecraft to produce the desired data return. The ABI and GLM instruments are designed to meet all performance requirements based on detailed interface specifications between instruments and the spacecraft, which will be presented in more detail in the following section. Unique to GOES-R, these requirements must be met during momentum unload events and 
station-keeping maneuvers. The GOES-R spacecraft is only allocated 120 minutes per year where the pointing and stability requirements can be exceeded [2][3]. This "operate-through" capability will provide unprecedented availability of observation data to the user community.

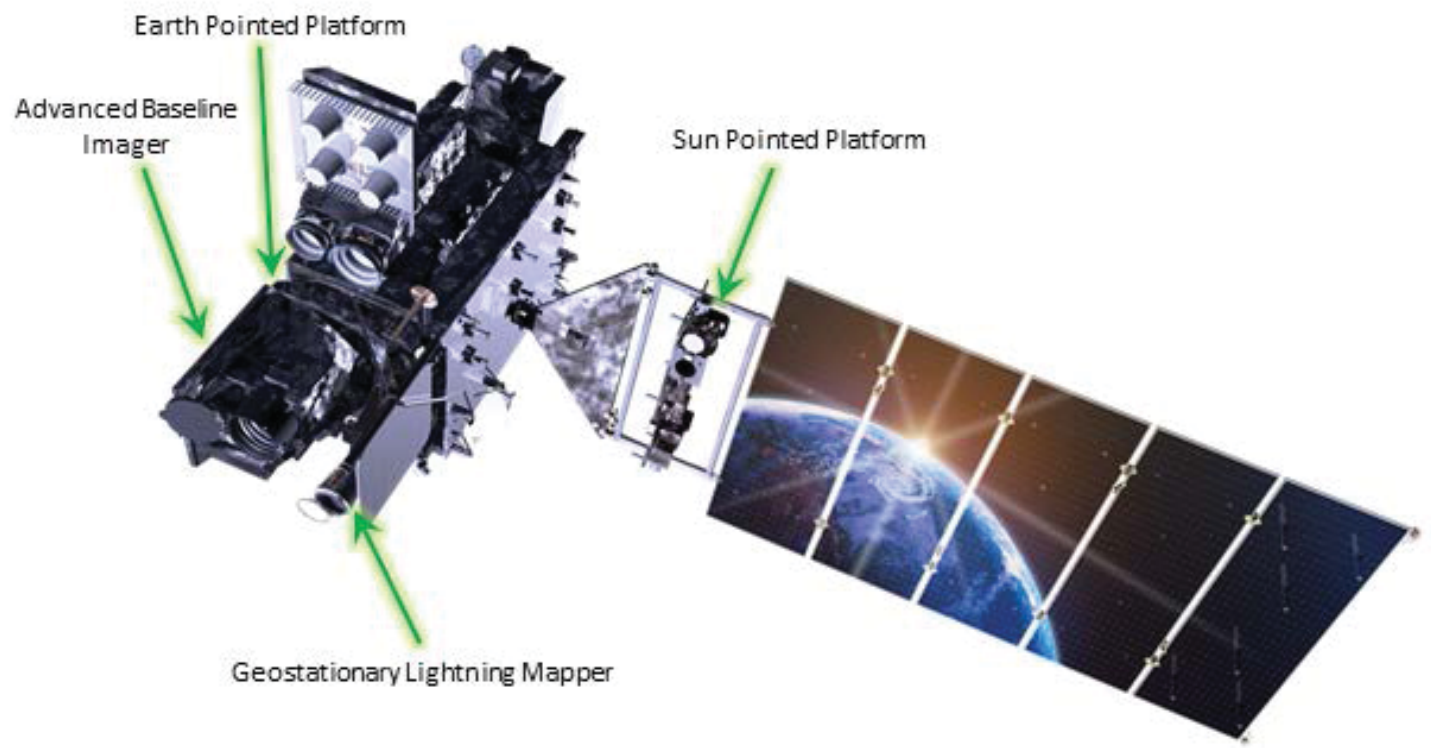

Fig. 1: GOES-R Spacecraft in Operational Configuration

In addition to Earth-observing instruments, GOES-R spacecraft also hosts two solar staring instruments on its Solar Pointed Platform (SPP), a particle detection instrument, and a magnetometer. These instruments have highly demanding pointing and stability requirements of their own. However, in this paper we focus on the pointing requirements of the Earth-observing instruments, and the performance of the GOES-R spacecraft in achieving those requirements.

We first present the attitude knowledge, orbit knowledge, pointing control, and pointing stability performance needed to realize the ambitious Image Navigation and Registration (INR) [4][5] objectives of GOES-R. We then discuss the design features implemented on the GOES-R spacecraft to achieve these performance requirements. Finally, we present the simulation predictions for the Attitude Determination (AD), orbit determination, pointing control, pointing stability, and jitter performance of the GOES-R spacecraft. The results show that the GOES-R design is successful in achieving the demanding requirements necessary to accomplish the mission.

\section{GOES-R GN\&C PERFORMANCE REQUIREMENTS}

The increased spatial, spectral and temporal resolution of the GOES-R Earth-observing instruments impose extremely demanding performance requirements on the spacecraft Guidance Navigation and Control (GN\&C) design, including attitude knowledge, Integrated Rate Error (IRE), orbit knowledge, pointing, pointing stability, and jitter. Customer defined GN\&C requirements in these areas [3] are summarized in Table 1.

The availability requirements mean these specifications must be met nearly continuously throughout the year. Freesland et al. [2] explored the GN\&C impacts associated with increasing operational availability by more than a factor of 30 over the current GOES-N series of spacecraft. They looked at existing technologies that could be employed to reduce total science outage resulting from daily momentum management, frequent East/West Station-Keeping (EWSK) and North/South Station-Keeping (NSSK) maneuvers, and twice yearly yaw flip maneuvers. Many of the features from that study are incorporated into the current GOES-R design. By meeting the operate-through requirements on pointing and pointing stability, GOES-R instruments will continue nominal operations during these maneuvers without any loss of fidelity.

The GOES-R attitude knowledge requirements are primarily driven by the instruments. The spacecraft GN\&C is required to provide inertial attitude knowledge to the instruments as a time-tagged attitude quaternion at $1 \mathrm{~Hz}$. 
The spacecraft is also required to provide low-latency gyro data to the $\mathrm{ABI}$ instrument at $100 \mathrm{~Hz}$. The ABI instrument uses this knowledge to provide real-time control of its Line-of-Sight (LOS). The instrument propagates its own attitude knowledge to achieve the GOES-R INR performance [6]. For other instruments, the attitude knowledge information is used during the post-processing on the ground. The stringent attitude knowledge requirements shown in Table 1 drive the spacecraft design to co-locate the Earth-observing instruments with the spacecraft Inertial Measurement Units (IMUs) and star trackers on the EPP.

Table 1: Customer Defined Level III GN\&C Requirement Summary

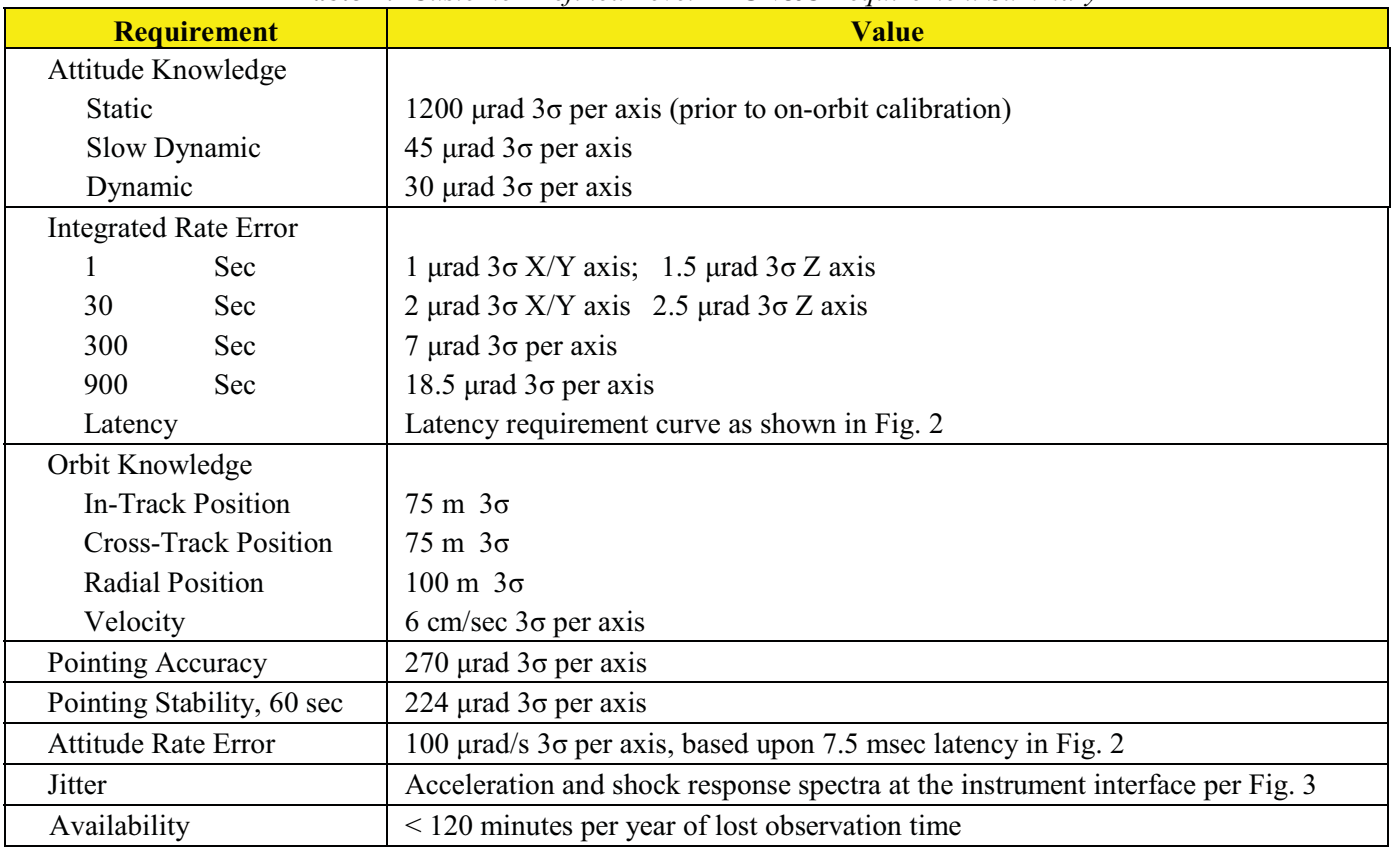

For the $100 \mathrm{~Hz}$ gyro data provided to ABI, the GOES-R spacecraft has a unique set of specifications on the estimated angular rates. Rather than specifying the rate errors in terms of traditional Farrenkopf gyro model parameters (angle white noise, angle random walk, and rate random walk) the project has defined performance in terms of IRE, which defines the accuracy of the integrated rate data over various intervals of time. The time intervals are directly related to instrument co-registration, swath-to-swath, star acquisition intervals, and instrument scan repeat times.

Since ABI uses the rate data for real-time LOS control, bandwidth and latency are also important. The project has set a minimum $-3 \mathrm{~dB}$ gyro bandwidth of $25 \mathrm{~Hz}$ for the gyro rate data. The latency requirements for the gyro rate data are redefined in terms of phase delay. The spacecraft phase delay requirement includes all delays from the time a physical event occurs through the time the data recording the event is received at the instrument. It includes sensor dynamics, sensor delays, spacecraft processor delays and transmission delays. The project phase delay requirement is cast in terms of a trade between spacecraft phase delay and allowable rate error as shown in Fig. 2. From this curve, spacecraft design has selected $7.5 \mathrm{msec}$ as the maximum phase delay for the gyro data provided to ABI. This results in a maximum allowable attitude rate error of $100 \mathrm{micro}-\mathrm{rad} / \mathrm{s}$, as shown in Table 1.

The spacecraft GN\&C is also required to provide orbit position and velocity to the instruments at a $1 \mathrm{~Hz}$ rate. Position accuracy requirements are driven by pixel navigation performance, and rate accuracy requirements are driven by the need to propagate orbit position between the 1 second updates. Traditional methods of providing orbit knowledge would be cumbersome and labor-intensive to achieve the requirements shown in Table 1. Instead, GOES-R has elected to implement an on-board Global Positioning System (GPS) receiver to provide orbit data with the specified accuracy. Because GOES-R is a geostationary satellite, this involves tracking extremely low level signals while operating above the GPS constellation. Inclusion of onboard GPS tracking for orbit determination is a new paradigm for GOES operations, and holds the promise of eliminating the need for active ranging and daily ephemeris uploads by the ground segment. 


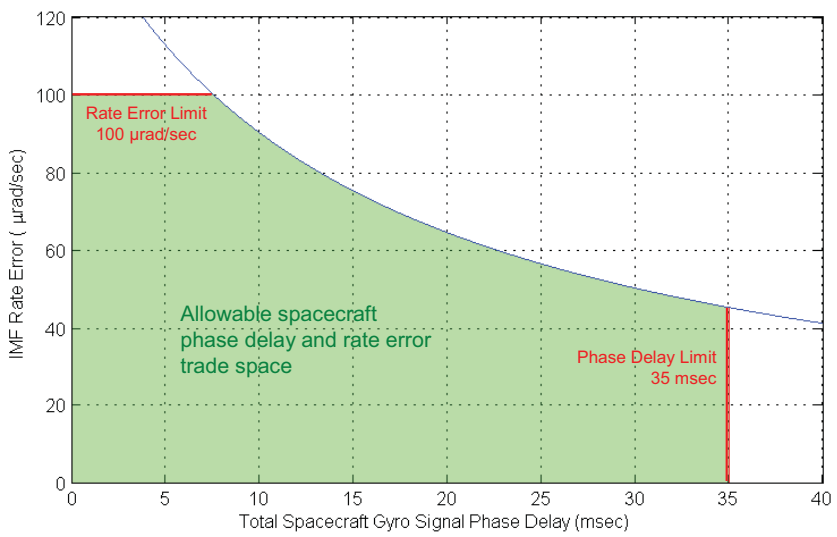

Fig. 2: Total spacecraft gyro signal phase delay and rate error trade space.

The GOES-R pointing and pointing stability requirements are comparable to other precision-pointing missions. However, these requirements apply during spacecraft maintenance events, such as momentum unloads and station-keeping maneuvers. This "operate-through" capability is unique to the GOER-R vehicle. Within the control design, there are a number of feed-forward paths to facilitate this operate-through capability. For example, the ABI instrument provides to the spacecraft predictions of the disturbance forces and torques created by ABI mirror motion for use in feed forward compensation. Freesland et al. [7], demonstrated the effectiveness of using this Predicted Interface Force and Torque (PIFT) signal to reduce attitude stability performance by a factor of 5 .

Jitter requirements for the nadir pointed instruments have been cast in terms of the linear translational accelerations and shock response spectra (SRS) at the instrument interfaces, as shown in Fig. 3. The spacecraft must achieve its requirement in the presence of the ABI disturbance shown. The "hats" in the acceleration and SRS profiles are due to the cryo-coolers in the ABI instrument. As can be seen from the plots, this does not leave much room for spacecraft-induced disturbances, such as reaction wheels and gimbal drive mechanisms. The requirements cover a broad frequency range out to $512 \mathrm{~Hz}$, which drives the EPP optical bench to be a stiff design, and drives the EPP to be passively isolated from the spacecraft bus.

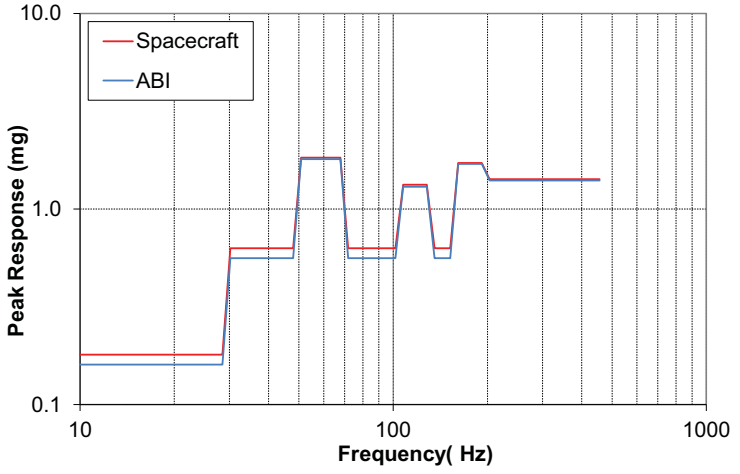

a) Linear translation acceleration limits.

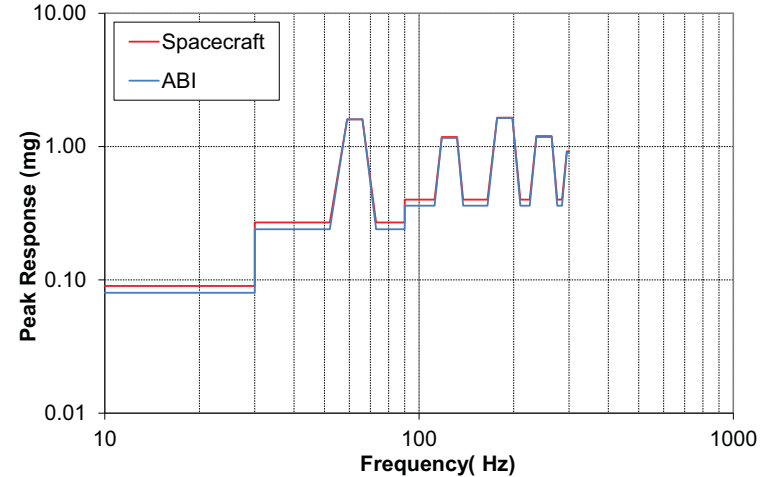

b) Shock response spectra limits.

Fig. 3: On-orbit jitter requirements are cast in terms of acceleration and shock response spectra at the instrument interface.

\section{GOES-R GN\&C DESIGN APPROACH}

As discussed in the previous section, the stringent GOES-R attitude determination requirements dictate that the IMUs and star trackers be co-located with the Earth-observing instruments, ABI and GLM. The resulting configuration is shown in Fig. 4, where the placement was driven by the instruments' field of regard, and the star tracker keep-out zones. GLM is a static staring instrument with no capability to compensate for alignment biases or shifts. Once GOES-R is on-orbit in the operational configuration, the GLM line-of-sight will be 
pointed at nadir. With its scanning mirrors, ABI has the capability to compensate for any offset between the $\mathrm{ABI}$ and GLM lines-of-sight.

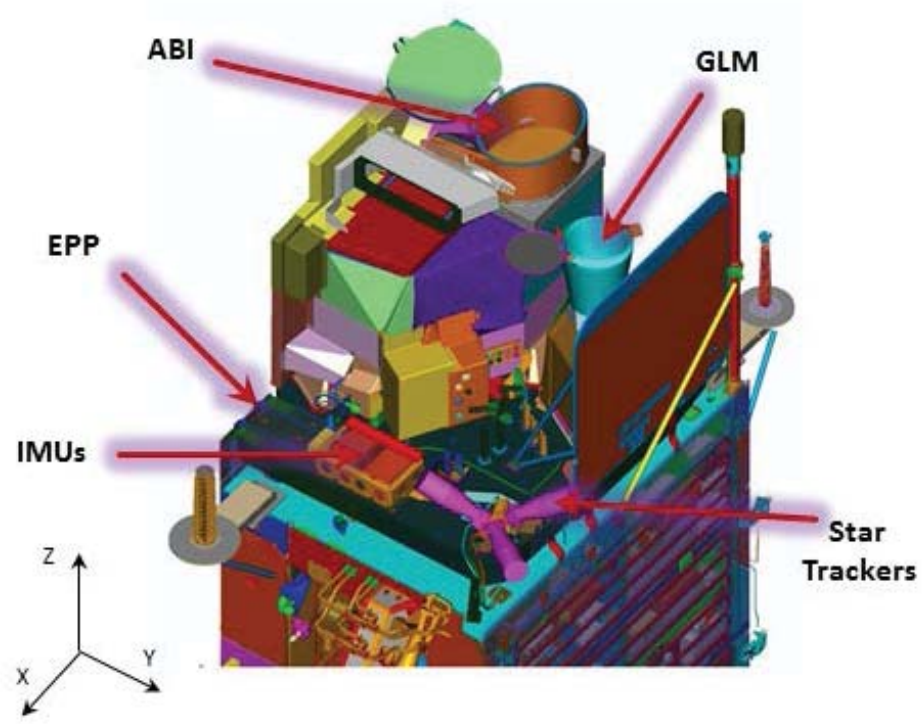

Fig. 4: Earth Pointed Platform Configuration for the GOES-R Spacecraft

To minimize the alignment shifts between the Earth-observing instruments and the GN\&C attitude determination sensors, they are all mounted to a common optical bench, the EPP. The EPP is constructed of carbon fiber facesheets over aluminum honeycomb. It spans approximately $2 \times 2 \mathrm{~m}$, and has a thickness of about $15 \mathrm{~cm}$. To meet the interface requirements presented in the previous section, the EPP is designed as a stiff structure with the first structural mode at approximately $50 \mathrm{~Hz}$.

The high stiffness of the optical bench readily transmits disturbances through the structure. To attenuate high frequency disturbances to the Earth-observing instruments from the spacecraft bus, which include reaction wheel disturbances, gimbal disturbances, and disturbances from the sun-pointed instruments, the EPP is passively isolated from the spacecraft bus with Honeywell D-Strut isolators [8] arranged in a modified Stewart platform configuration. The isolation system provides attenuation for frequencies above $\sim 5 \mathrm{~Hz}$ in all six degrees-of-freedom.

The GOES-R spacecraft design utilizes 6 Honeywell HR-18 reaction wheels, arranged as shown in Fig. 5. The use of 6 wheels provides functional redundancy, low-speed wheel operation (which reduces vibration), and relatively high torque capability.

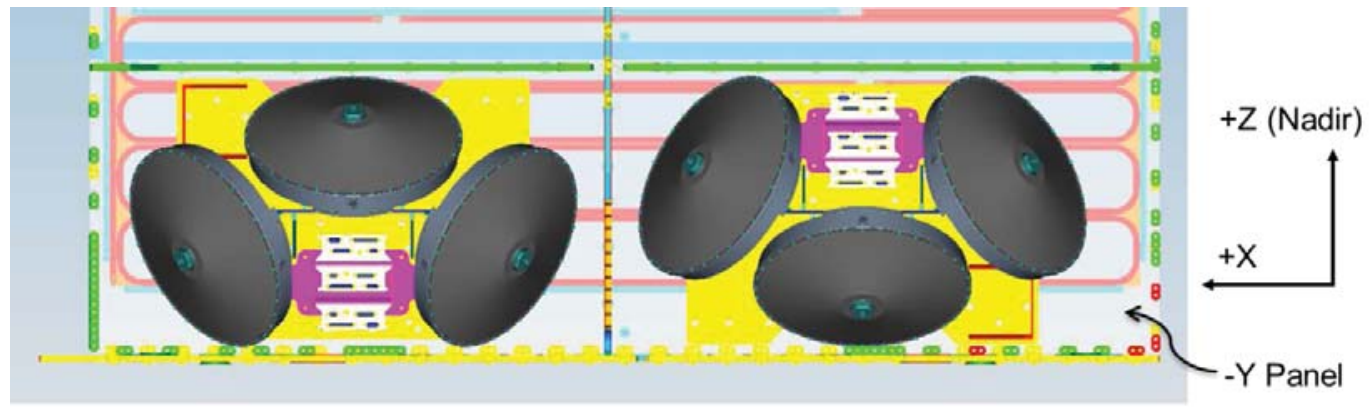

Fig. 5: Reaction Wheel Configuration of the GOES-R Spacecraft

Analysis of this design has shown that high frequency induced vibration from the reaction wheels can still be observed on at the instrument mounting interface, even with the EPP isolation system. To address this, a secondary passive isolation system manufactured by Moog/CSA Engineering was introduced for each reaction 
wheel. The design is based upon Moog's Visco-Elastic Material (VEM) vibration isolation technology [9]. This secondary isolation system provides attenuation for frequencies above $\sim 50 \mathrm{~Hz}$ for each wheel. The performance for jitter control of this configuration will be discussed further in Section 7.

The "operate through" requirement for GOES-R drove the development of Aerojet Rocketdyne's miniature $0.08 \mathrm{~N}$ Low Thrust REA (LTR) for use during Momentum Adjust (MA) maneuvers and EWSK maneuvers. The small thrust from the LTR's can be balanced by reaction torque from the reaction wheels, which allow continuous firing of the LTRs with minimal spacecraft attitude disturbance. Because of their high Isp, $0.2 \mathrm{~N}$ arcjets (also built by Aerojet Rocketdyne) are used for NSSK. As with the LTR's, the small thrust from the arcjets can be balanced with torque from the reaction wheels, and the attitude excursions during NSSK maneuvers remain within pointing requirements. The pointing and pointing stability performance during these maneuvers will be discussed in more detail in Section 6.

To reduce the impact of low frequency disturbances, and to increase the low-frequency gain of the attitude controller, GOES-R includes Active Vibration Damping (AVD) developed by Lockheed Martin [10]. This additional compensation phase stabilizes the first several structural modes, up to $\sim 2 \mathrm{~Hz}$ in the GOES-R implementation. Because GOES-R is a large single-wing spacecraft design, the lowest structural modes limit the control authority that can be achieved using traditional design methods. The first structural mode is at $\sim 0.25$ $\mathrm{Hz}$, and is related to the large solar panel flexibility coupled with the deployment hinge stiffness. AVD compensation is particularly effective for the first three structural modes of GOES-R.

Finally, the gimbal design used for the azimuth and elevation control of the solar array and SPP incorporates the proven low-disturbance design first implemented on the Mars Reconnaissance Orbiter [11]. The design is based upon a zero-backlash harmonic drive with a relatively high gear reduction of 200:1. The low disturbance capability is provided by a 2-phase brushless motor driven by a sine drive commutation, which effectively eliminates motor cogging. Additionally, a high bandwidth rate-loop is implemented on the motor rate, which essentially eliminates most of the harmonic drive friction and nonlinear effects. As shown in Section 6, the high performance of the gimbals removes gimbal dynamics as a significant pointing disturbance source.

\section{ATTITUDE DETERMINATION PERFORMANCE}

For attitude determination, GOES-R utilizes a Northrop Grumman Scalable Space Inertial Reference Unit (SSIRU), and a SODERN Hydra Star Tracker with three optical heads. The SSIRU gyro data are sampled at $200 \mathrm{~Hz}$, and the star tracker data are sampled at $20 \mathrm{~Hz}$. Attitude estimation is performed using a kinematic 6state extended Kalman filter [12], which combines quaternion outputs from the star tracker with angular rate measurements from the SSIRU to produce a 3-state attitude error estimate and 3-state gyro bias error estimate. As with previous GOES satellites, accurate attitude and rate estimates are critical to INR requirements as they are used in the ground-based motion compensation. For GOES-R, rate estimates are also used for real-time ABI mirror control to steer out jitter up to the first instrument mode [6]. In the GOES-R attitude determination implementation, the star tracker measurements and SSIRU measurements are synchronized with the spacecraft control frame to provide the most accurate attitude estimate possible.

The SSIRU plays a key role in meeting the GOES-R INR requirements. High bandwidth, low-latency rate measurements are critical for accurate motion compensation and real-time mirror control for the ABI instrument. The IRE requirements specify how much error can be accumulated when integrating measured gyro rates. As shown in Table 1, IRE requirements are specified over different time windows from 1 to 900 seconds. The 1 second window is completely driven by gyro performance, particularly angle white noise. The other windows are driven by a combination of gyro performance, Kalman filter bias estimation, and stability of the mounting interface. The SSIRU was selected for GOES-R because of the high bandwidth and low latency of the RS-422 gyro data output, as well as the low-noise characteristics of the four hemispherical resonator gyros.

The three-head SODERN Hydra Star Tracker is used for attitude measurements, with two heads operating continuously and one serving as a cold spare. The Hydra design provides the capability to synchronize each star measurement with an externally provided $20 \mathrm{~Hz}$ reference signal. Star measurements from the multiple heads are combined within the star tracker software by rotating the measurements into a common reference frame and applying a QUEST optimization [13] to produce a "fused" attitude estimate from the 2 optical heads [14]. For the GOES-R star tracker geometry and geostationary orbit, Fig. 6 shows typical measurement errors from the fused attitude solution operating with two optical heads. Each head can track up to 15 stars at $20 \mathrm{~Hz}$, so the 
QUEST optimization is performed on up to 30 star measurements, depending on stray light conditions and the sky location being imaged by each optical head. This is shown in the bottom subplot of Fig. 6 .
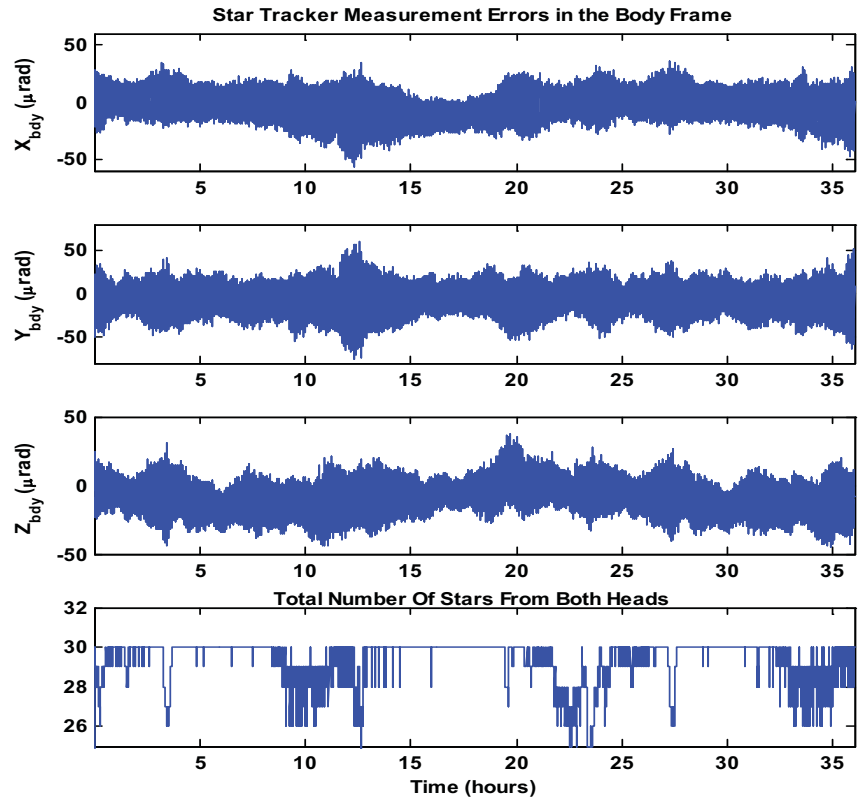

Fig. 6: HYDRA Star Tracker Measurement Errors

The 6-state Kalman filter does not include any estimation of the alignment errors between the SSIRU and the star tracker. Instead, a calibration sequence will be performed during the initial on-orbit checkout to remove gyro to star tracker alignment errors. The calibration sequence will also estimate the gyro scale factor errors. The expected residual alignment and scale factor errors from the calibration sequences are included in the AD performance simulation results shown below.

Attitude estimation performance has been evaluated using worst-case GOES-R time-domain simulation profiles for attitude and attitude rate of the SSIRU and star tracker mounting interfaces. These profiles were derived from the high-fidelity pointing and jitter simulation results for worst-case vehicle disturbances. The rate input profile used to drive the SSIRU model is shown in Fig. 7. The effect of ABI instrument operations is clearly evident in the data, particularly in the $\mathrm{X}$ and $\mathrm{Z}$ axes (the blue and red curves, respectively). The nominal orbital rate can be seen in the $\mathrm{Y}$-axis (the green curve).

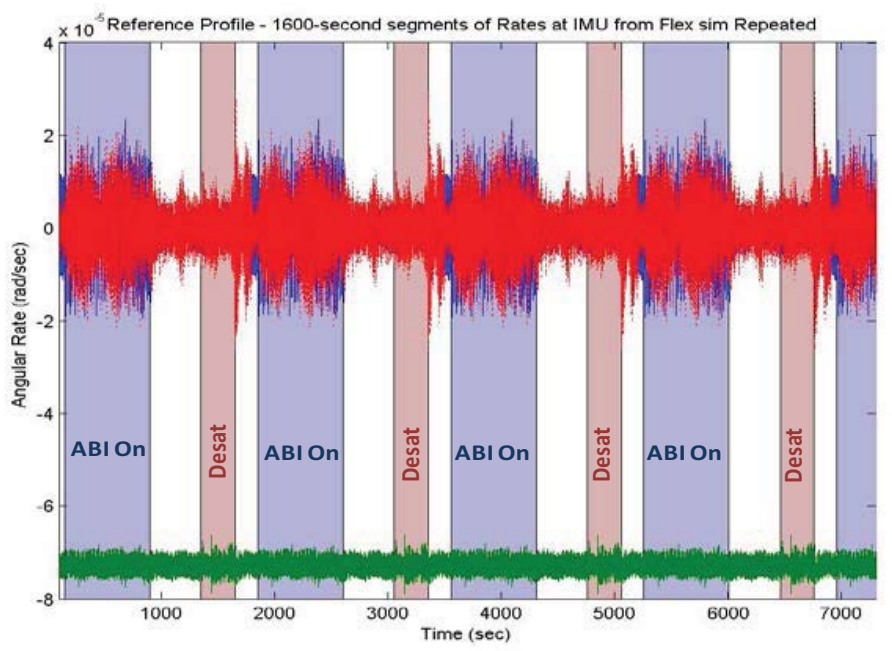

Fig. 7: AD Simulation Input Rate Profile 
The attitude and attitude rate profiles were used as inputs to high-fidelity star tracker and SSIRU models, and the outputs of these models were in turn used as inputs to the attitude determination flight software. The simulation is implemented in Simulink and run in with Monte Carlo dispersions of key error sources. The attitude and bias estimation results from the Monte Carlo simulations are shown in Fig. 8. The attitude estimation performance is seen to meet the requirement suballocations shown in Fig. 8 for attitude knowledge.
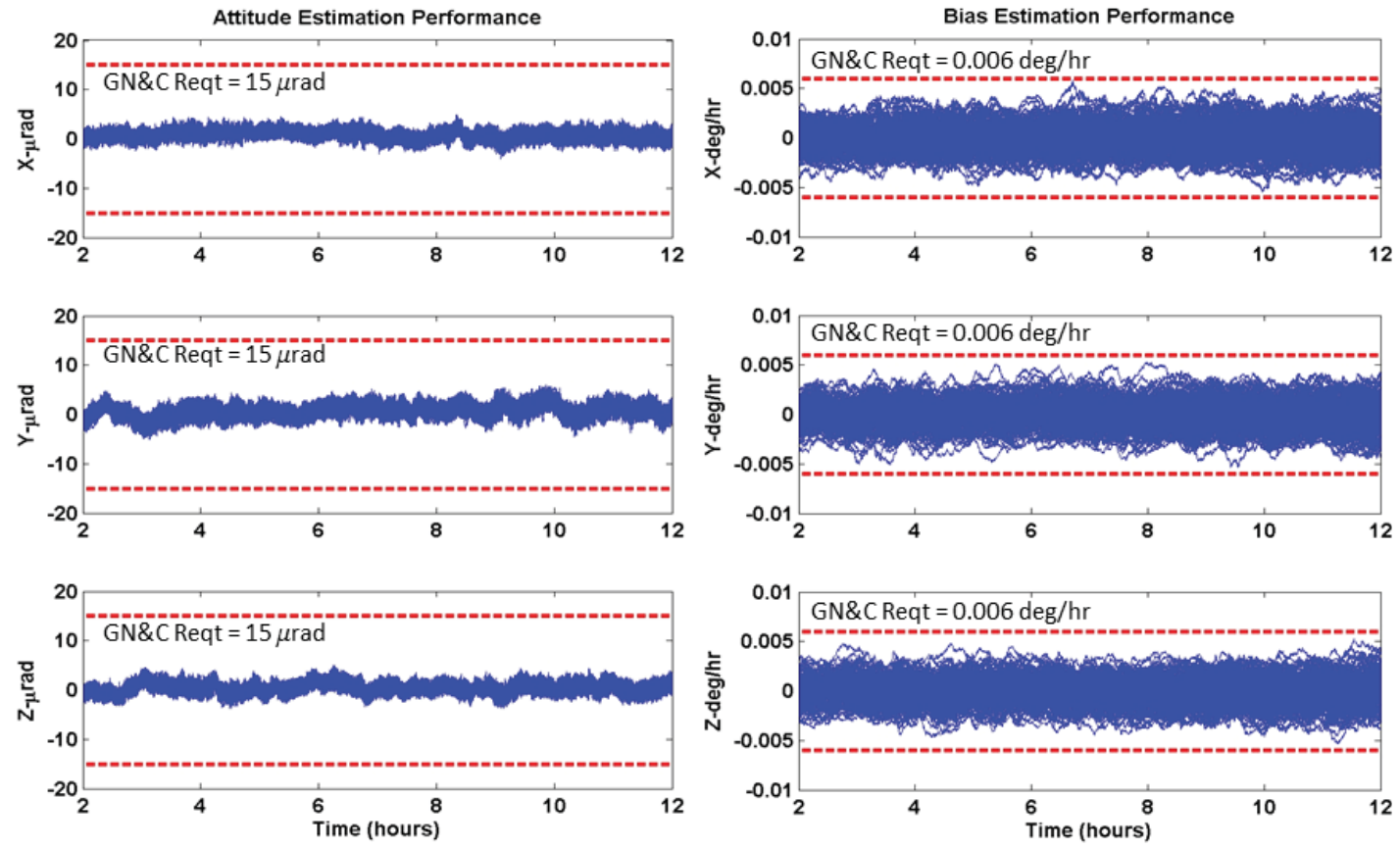

Fig. 8: Attitude and Bias Estimation Errors from Attitude Determination Monte-Carlo Simulations

The IRE requirements are the most unique AD performance requirements for GOES-R. To establish the performance characteristics of the gyros, 24 hours of $200 \mathrm{~Hz}$ data from the gyros were collected with the GOESR SSIRU mounted to a granite block. Analysis of the gyro data has been performed to estimate the angle white noise, angle random walk, and rate random walk parameters for each gyro. The SSIRU model in the AD simulation was configured with these model parameters, and the attitude and attitude rate profiles discussed above were used as inputs to the AD simulation. The resulting IRE performance is shown in Fig. 9 for the four different IRE windows. As observed in the simulation results, IRE performance requirements are met with considerable margin for the GOES-R SSIRU.
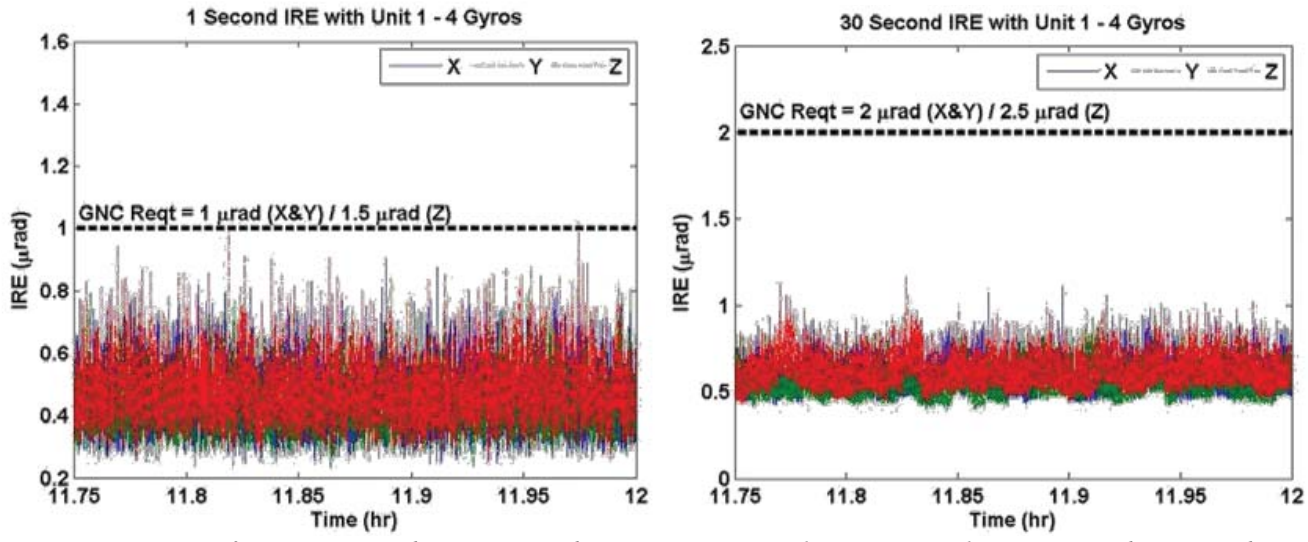

Fig. 9a: IRE Performance Based on Measured SSIRU Noise Levels in AD Simulation, $1 \mathrm{~s}$ and $30 \mathrm{~s}$ windows 

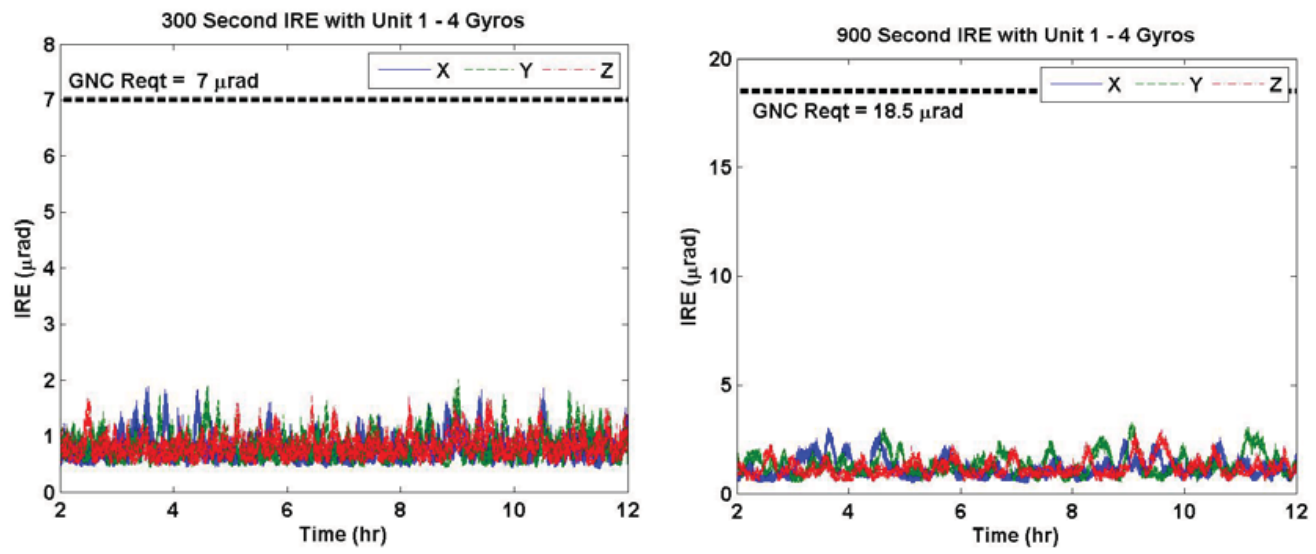

Fig. 9b: IRE Performance Based on Measured SSIRU Noise Levels in AD Simulation, $300 \mathrm{~s}$ and $900 \mathrm{~s}$ windows

\section{ORBIT DETERMINATION PERFORMANCE}

A key part of the GN\&C component suite for GOES-R is the upgraded Viceroy GPS Receiver (GPSR) from General Dynamics coupled with a GPS antenna designed by Lockheed Martin. The Viceroy-4 was developed specifically for the GOES-R program. The antenna design is tailored for operations in a Geosynchronous Earth Orbit (GEO). The new GPSR design and custom GEO antenna design enable onboard autonomous navigation, which is a critical enabling technology for this mission.

A GPS receiver at GEO altitude ( $\sim 35,786 \mathrm{~km})$ is $15,000 \mathrm{~km}$ farther away from Earth than the GPS constellation. Satellites comprising the GPS constellation are designed to transmit signals towards Earth. Therefore, a GEO spacecraft such as GOES-R must have its GPS receive antenna nadir pointing in order to receive the GPS signals that leak around the Earth, as shown in Fig. 10.

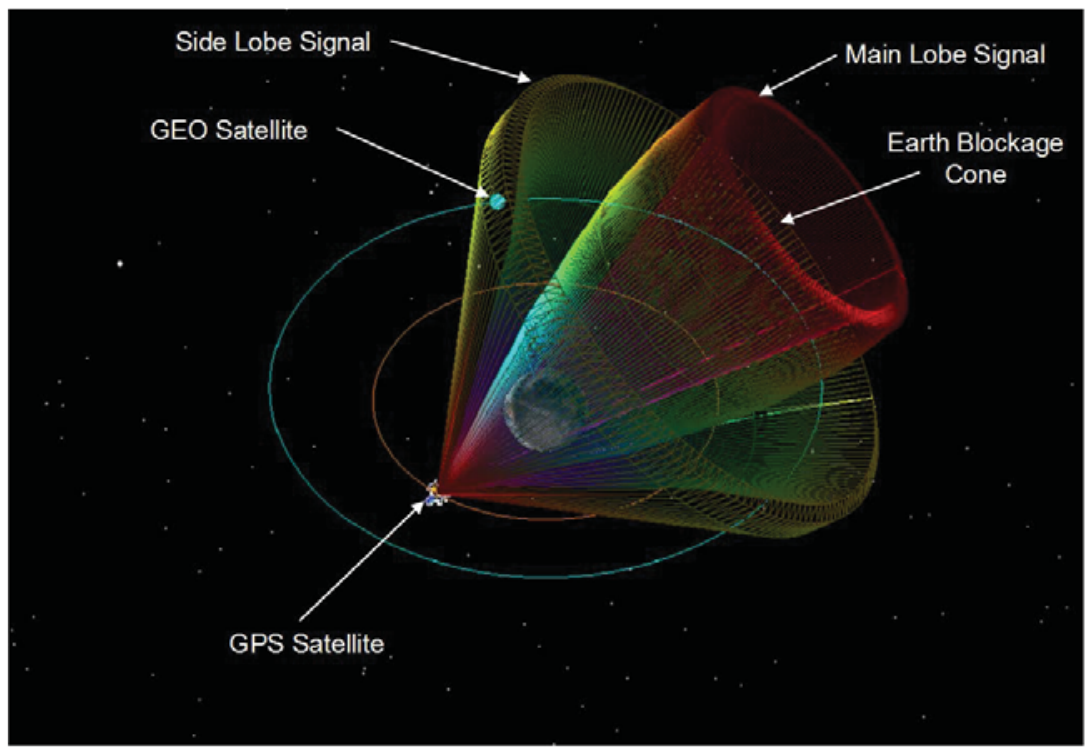

Fig. 10: GPS Signal as Seen by a Geostationary Satellite

A GPS receiver performs optimally when it tracks 4 or more GPS Satellites. Analysis shows that this cannot be achieved at GEO when only the GPS main lobe signals are used [15][16]. Because GPS side lobe signals are inherently weak, and because free space path loss at GEO is up to $10 \mathrm{~dB}$ compared with low Earth orbit, tracking GPS side lobes is extremely challenging. Furthermore, only main-lobe signal power is specified in the GPS system specification. The antenna designers for the various GPS vehicles (Block II, IIA, IIR, IIRM, and IIF) have chosen slightly different methods of meeting main-lobe power requirements. As a result, the side-lobe characteristics vary depending upon the specific GPS satellite in view, as illustrated in Fig. 11. A GPS receiver 
attempting to exploit side lobe information must have the dynamic range to distinguish a low power signal from the noise while not saturating when a high power signal is present [16].This characteristic of the GPS constellation makes analysis of GEO receiver availability particularly difficult.

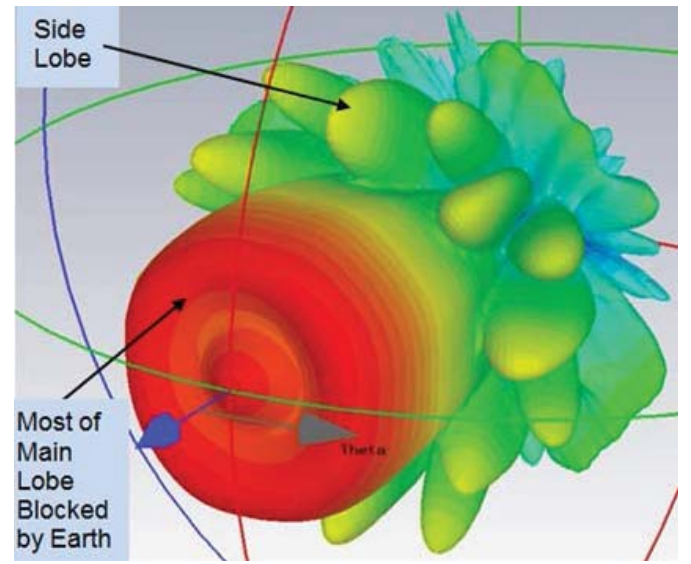

Fig. 11: GPS transmit antenna pattern in 3D illustrating side lobe structure detail

The orbit determination accuracy requirements are shown in Table 1. In order to verify that the GOES-R GPS navigation system can meet the stringent accuracy requirements during momentum unload events and stationkeeping maneuvers, a worst-case test scenario was developed for the GOES-R GPSR testing. The simulated GPS constellation was modified to establish a worst-case scenario. In this scenario, the fewest number of trackable GPS satellites occurs during the momentum unload events and station-keeping maneuvers. This highfidelity simulation also reflects the same GPS signal power profiles that the GPSR will see on-orbit [17]. Performance of the GPSR using this scenario during acceptance testing is summarized in Table 2. Position and velocity errors from a high-fidelity GEO simulation test case immediately following a GPSR cold-start are shown in Fig 12. Because of the high number of visible and tracked GPS satellites, the results indicate that most of the tracked GPS satellites are from using side lobe signals.

The onboard GOES-R spacecraft operations, including the attitude determination, orbit determination, and instrument operation, are all synchronized to the GPSR one pulse per second (1-PPS) clock output. As such, the accuracy and stability of this 1-PPS signal are critical. During the acceptance tests, the 1-PPS timing performance was also monitored during the 3-day GEO simulation. The 1-PPS error is required to be less than $250 \mathrm{~ns}$ over all environments. The timing error results are also summarized in Table 2.

Table 2: GOES-R GPSR Performance for Worst-Case Scenario GPSR Acceptance Test Results, Position Error (3-Day Scenario) 

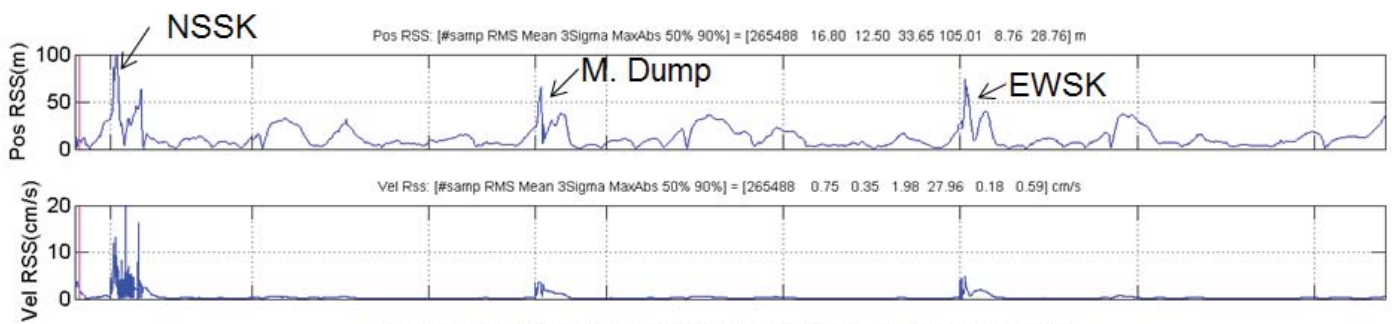

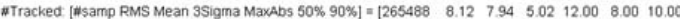
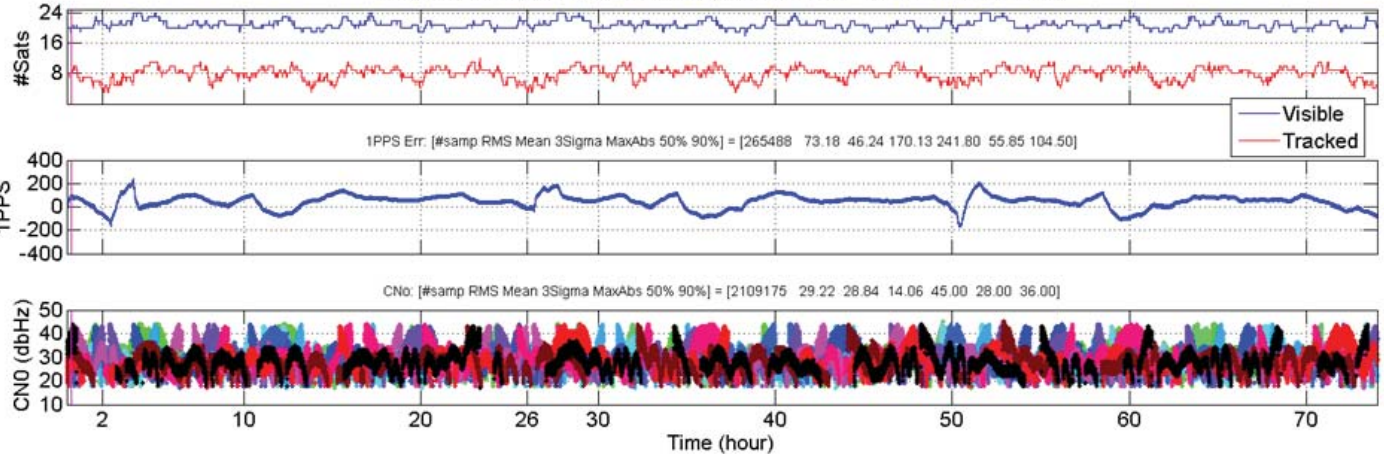

\begin{tabular}{|c|c|c|}
\hline \multirow{2}{*}{ Parameter } & Position $(\mathrm{m})$ & Velocity $(\mathrm{cm} / \mathrm{s})$ \\
\cline { 2 - 3 } & $3 \sigma$ & $3 \sigma$ \\
\hline RSS Error & 33.65 & 1.98 \\
\hline
\end{tabular}

Fig. 12: GPSR Position performance over 3 days

The time to initialize upon power up is a good indicator of the performance capability of a GPSR. Fig. 13 shows the time elapsed from power on for the GOES-R GPSR to track 4 satellites and obtain a position solution within specification. As observed in Fig. 13, the GOES-R GPSR acquires a position fix within 8 minutes for more than $95 \%$ of the cases.

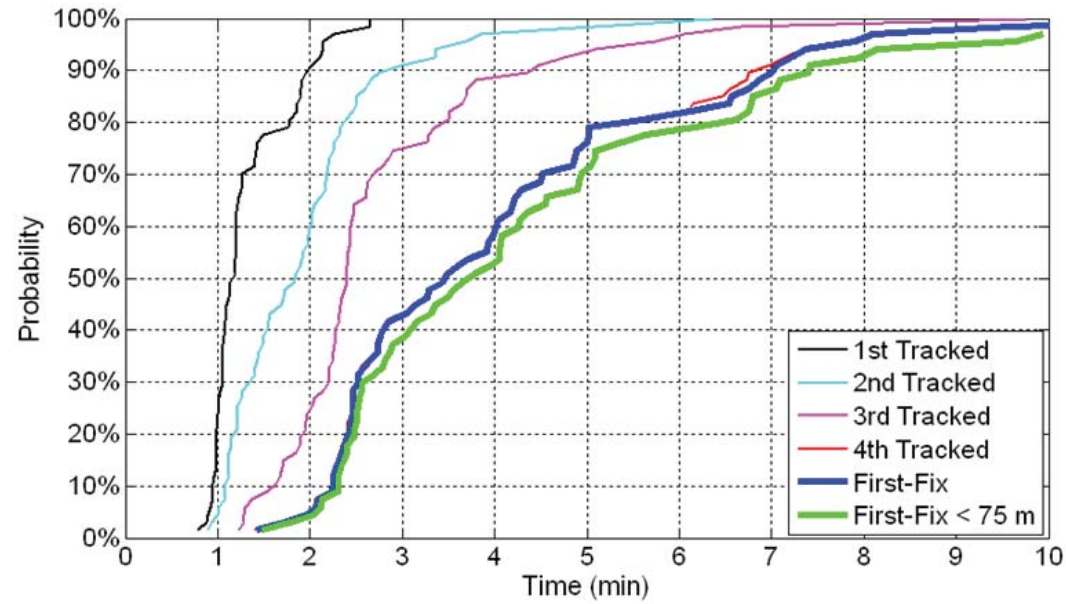

Fig. 13: GOES-R GPSR Qualification Test Data - Acquisition Time Performance of Viceroy-4

Once acquisition is attained, the Viceroy-4 outputs the spacecraft position and velocity in the Earth-Centered Earth-Fixed (ECEF) reference frame at $1 \mathrm{~Hz}$. The GPSR-provided ECEF position and velocity are converted to the International Celestial Reference Frame (ICRF) by the GOES-R onboard software. The ICRF position and velocity are converted to equinoctial elements, which are used to propagate the orbit at $20 \mathrm{~Hz}$ and to provide the nadir and orbit normal vectors needed by the attitude control system. 


\section{POINTING CONTROL AND POINTING STABILITY PERFORMANCE}

\section{A. WORST PAYLOAD POINTING CASE}

As discussed earlier, the GOES-R spacecraft is required to meet all instrument pointing requirements with less than 120 minutes per year of lost observation time. This means that the spacecraft must meet payload pointing requirements during all momentum unload and station-keeping events. These events are categorized into MA maneuvers, EWSK maneuvers, and NSSK maneuvers. MA maneuvers are used to adjust the momentum state of the spacecraft Reaction Wheel Assemblies (RWAs), EWSK maneuvers are used for orbit longitude control, and NSSK maneuvers are used for orbit latitude control. MA maneuver scenarios are the most stressful on the control system as they impart a comparatively large torque on the spacecraft to provide a rapid change in momentum state. By design, the EWSK and NSSK maneuvers apply relatively small torques to the spacecraft. The thrusters used for EWSK and NSSK maneuvers are fired in balanced pairs to minimize torque imparted on the spacecraft, and therefore produce smaller payload pointing error transients than MA maneuvers. Consequently, the MA maneuvers produce the largest payload pointing errors of any of the operate-through maneuver types.

A Matlab/Simulink tool developed at Lockheed Martin called the Momentum and Station-Keeping Simulation (MSKSim) provides moderate fidelity modeling of the RWA speeds throughout the GOES-R mission. The MSKSim models all MA, EWSK, and NSSK maneuvers during the 15 year spacecraft mission, both for storage and operational mission phases. Results from this simulation have been analyzed to determine which combination of LTRs during MA maneuvers result in the largest torques being imparted on the spacecraft. The resultant combination of thrusters, combined with worst case thruster performance assumptions and the worst case center of mass assumptions, represents the worst case pointing and pointing stability scenario. The details of this scenario are used as inputs to both the MSKSim and the high fidelity pointing/jitter simulation discussed in Section 7. Between these two simulation assets, a comprehensive picture of the GOES-R low frequency and high frequency pointing and pointing stability performance is obtained for the worst-case scenario.

\section{B. POINTING CONTROL AND STABILITY PERFORMANCE}

Common to all operational scenarios, the disturbances affecting low frequency pointing performance include solar array articulation, ABI scan mirror disturbances, and RWA friction, gyroscopic, and zero crossing disturbances. In addition to these common disturbances, momentum adjust cases also include LTR thruster disturbances (torque and thrust variation). Because many of these disturbances are deterministic and predictable, GOES-R Flight Software (FSW) uses feed forward capabilities to improve pointing stability. The gimbal articulation controller uses spacecraft body rate estimates as a feedforward term to steer out spacecraft body motion for the sun pointed instruments. For the Earth-observing instruments, ABI scan mirror disturbances are mitigated through feedforward of the ABI PIFT data from the instrument. Torques produced by the LTRs and arcjets are predicted and fed forward as compensation for those disturbances. Gyroscopic torques due to the spinning RWAs are also fed forward through the attitude controller. All of these feedforward sources are modeled in the high fidelity pointing and jitter simulation. Along with feedforward prediction algorithms, RWA friction and zero crossing disturbances are mitigated by the implementation of a wheel speed controller, which acts on the error between the commanded speed and the actual wheel speed estimate. Due to the relatively low number of pulses per revolution from the RWA digital tachometers, low speed quantization effects are mitigated in the RWA control algorithm through inclusion of a model based speed estimator. Finally, FSW and the high fidelity simulation both incorporate the AVD algorithm, which adds significant damping to low frequency ( $\leq$ $1 \mathrm{~Hz}$ ) spacecraft structural modes and increases the low-frequency gain of the attitude control loop.

The GN\&C high fidelity pointing and jitter simulation is used to simulate the time domain performance of the spacecraft during nominal and operate-through operations. Results presented herein represent a stacked worstcase momentum adjust scenario with the worst-case LTR set providing the largest possible torque on the spacecraft. The simulation includes attitude/rate error generation and control, AVD, RWA wheel momentum control, articulation control, and hardware interface algorithms for the RWAs, IMUs, and thruster disturbance feedforward. Hardware models incorporated in the simulation include an RWA model (with friction, ripple, induced vibration disturbances and digital tachometer models), an IMU model, solar array gimbal models (with motor, harmonic drive, slip ring, and trailer bearing models), an LTR model, and an arcjet model. Other disturbances in the model include gyroscopic torques and instrument disturbances (ABI scan mirrors, cryocoolers, and solar calibration cover). The spacecraft dynamics model is a linearized state space model generated from a multi-body flexible spacecraft model assembled in the MSC ADAMS software package. This is the 
same model used in the jitter performance simulation presented in Section 7, except the MSKSim truncates the spacecraft modal content to $25 \mathrm{~Hz}$. Whereas the jitter performance simulation retains spacecraft modal content up to $600 \mathrm{~Hz}$, the MSKSim addresses performance at lower frequency and therefore does not require modes beyond $25 \mathrm{~Hz}$.

As shown in Fig. 14 and Fig. 15, the spacecraft meets all pointing control and stability requirements during this stacked worst-case scenario. It should be noted that the requirements shown on these plots are smaller than the numbers that appear in Table 1. The attitude control requirement in the attitude error plot (100 micro-rad, 3sigma, per axis) is the suballocation of the overall pointing requirement to the attitude controller. Attitude estimation errors are not included in the attitude error plot of Fig. 14, as those errors have a separate suballocation. Of course, both the attitude control error and the attitude estimation error contribute to the overall pointing error.
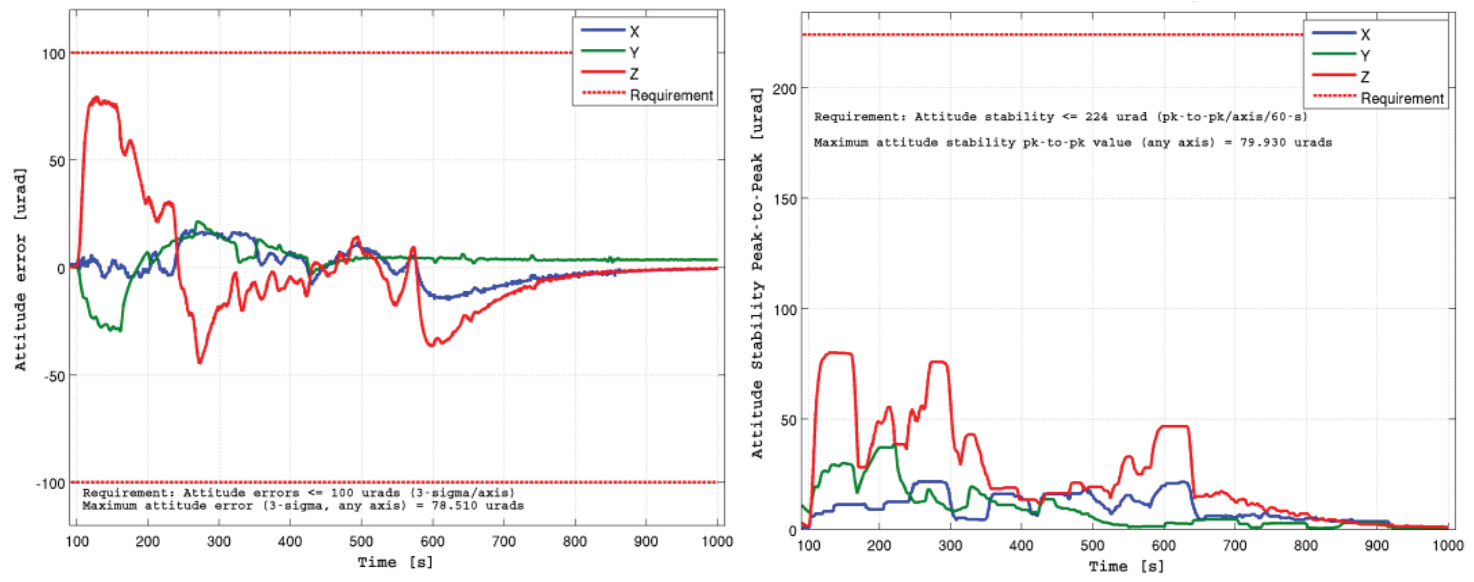

Fig. 14: Attitude Control and Attitude Stability Performance of the GOES-R spacecraft for the Worst-Case OperateThrough Scenario.

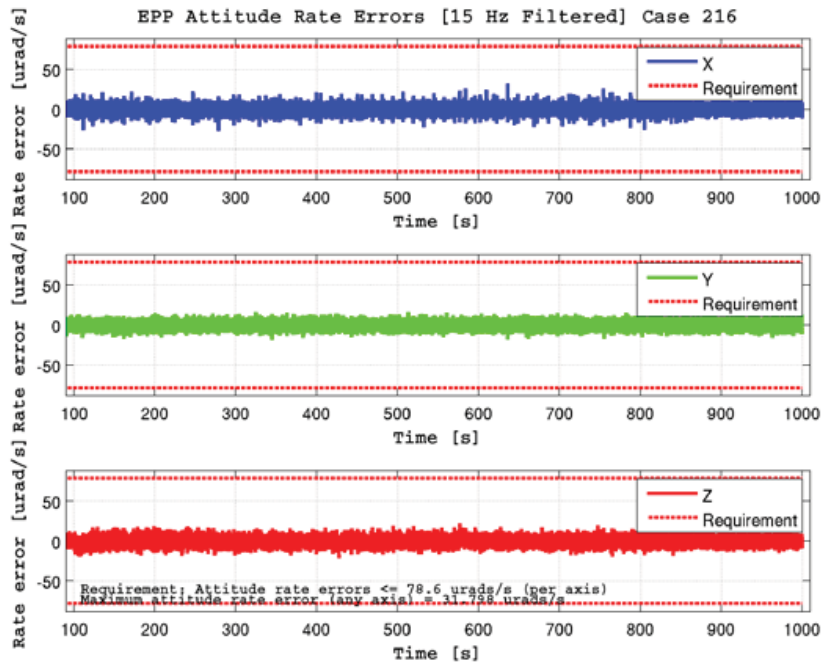

Fig. 15: Attitude Rate Error Performance of the GOES-R Spacecraft for the Worst-Case Operate-Through Scenario.

\section{JITTER PERFORMANCE}

High-frequency jitter refers to disturbances in the $10 \mathrm{~Hz}-600 \mathrm{~Hz}$ frequency range, and the spacecraft attitude control system response is virtually open-loop at such high frequencies. The highest control system bandwidth of all the GOES-R controllers, the AVD loop, is less than $2 \mathrm{~Hz}$, which is below the frequency of the jitter response threshold. Analyses and simulations have been completed to predict GOES-R jitter and instrument LOS performance, and to identify worst-case scenarios that produce upper-bound performance limits. The 
results are compared with the GOES-R requirements discussed in Section 2, and are compared with additional LOS guidelines provided by the customer. Because of the lengthy simulation runtimes associated with these predictions, analyses have focused upon developing worst-case disturbance inputs as forcing functions for the simulation.

\section{A. DYNAMIC MODELING APPROACH FOR JITTER ASSESSMENT}

The results presented here were generated using the closed-loop GN\&C high-fidelity jitter simulation with spacecraft dynamic models having frequency content up to $600 \mathrm{~Hz}$. The jitter simulation includes structural models of the spacecraft bus, appendages, EPP isolation, reaction wheels, reaction wheel isolation, and instruments. The structural models are all derived from NASTRAN representations, but only the modes up to $600 \mathrm{~Hz}$ are retained. Conservative models are used for the damping of the structural modes, with the damping set to be $0.002(0.2 \%)$ for modes less than or equal to $50 \mathrm{~Hz}$, and $0.004(0.4 \%)$ for modes greater than $50 \mathrm{~Hz}$. To accurately capture the modal responses, the simulation is integrated at $8 \mathrm{kHz}$, and key outputs are recorded at $2 \mathrm{kHz}$.

The high-fidelity jitter simulation includes extensive modeling of disturbance sources on the GOES-R spacecraft. Incorporated are high fidelity models of the reaction wheels (motor ripple, static and dynamic imbalance, bearing eccentricity, induced vibration), gimbals with harmonic drive disturbances, fuel and oxidizer slosh, ABI scan mirror motion, and ABI cryo-coolers. High-frequency disturbances dominate the GOES-R spacecraft jitter and LOS performance. Only the reaction wheel induced vibration and ABI cryo-cooler produce significant disturbances at high frequency. Lower frequency disturbances, such as reaction wheel zero-speed crossings, gimbal drive responses, slosh disturbances, and thruster noise and bubble events, can be observed in the jitter and LOS performance, but they are relatively small contributors.

The jitter simulation also incorporates high-fidelity models of the GOES-R control law implementation. Sensor noise models, computational delays, quantization effects, control uncertainties, and prediction errors for feedforward terms are all included. All of the GOES-R control loops are active for these simulations, including articulation control, active vibration damping, feed-forward compensation for the ABI scan mirror disturbances, and feed-forward compensation for thruster firings,. As discussed in the previous section, MA maneuvers are the most stressing disturbances for pointing control and pointing stability. For these jitter simulations, the MA software commands the 6 wheels from initial speeds of $\left[\begin{array}{lllllll}+1100 & +1100 & 0 & -1100 & -1100 & 0\end{array}\right]$ RPM to final wheel speeds of $\left[\begin{array}{llll}-1100 & 0+1100+1100 & 0 & -1100\end{array}\right]$ RPM. This covers the operational range of reaction wheel speeds for the mission, while also introducing thruster disturbances into the simulation response.

As shown in the results to follow, the ABI cryo-coolers dominate the jitter response. Special consideration has been paid to the accurate modeling of the cryo-cooler disturbances. The ABI cryo-coolers each contain a compressor (integral unit) and a remote unit which produce 3 -axis force disturbances. These disturbances are modeled using a Fourier series expansion of the first eight harmonics per axis. In these simulations, each harmonic for each axis starts with random phase set by a random number seed, which allows repeatable behavior from run to run. Magnitudes of each of the harmonics for each axis were provided by the ABI instrument supplier. The cryo-cooler disturbance forces are applied at the finite element model nodes for the $\mathrm{ABI}$ cryo-cooler. The cryo-cooler responses have been tuned to match ground test data from the ABI instrument ground testing.

\section{B. EVALUATION OF GOES-R ACCELERATION REQUIREMENTS}

Fig. 16 shows the acceleration bandpass requirements, the SRS requirements, and the jitter response at the ABI mounting interface. There are two different requirement curves for the acceleration bandpass and the SRS responses: GIRD157 and GIRD1110 refer to the specifications provided to the instrument, whereas GNC212 and GNC215 refer to the suballocations given to the spacecraft. The differences between the curves represent customer reserve or margin that can be applied to either side of the spacecraft/instrument interface. As shown in the plots, the bandpass and SRS interface acceleration requirements for the ABI instrument are satisfied with large margins when ABI is not operating. The RWA induced vibration disturbances are the primary disturbance sources active for these simulation cases. These disturbances are well attenuated by the RWA isolation and the EPP isolation. Although not shown, the interface acceleration requirements for the GLM instrument have similarly large margins when $\mathrm{ABI}$ is not operating. 

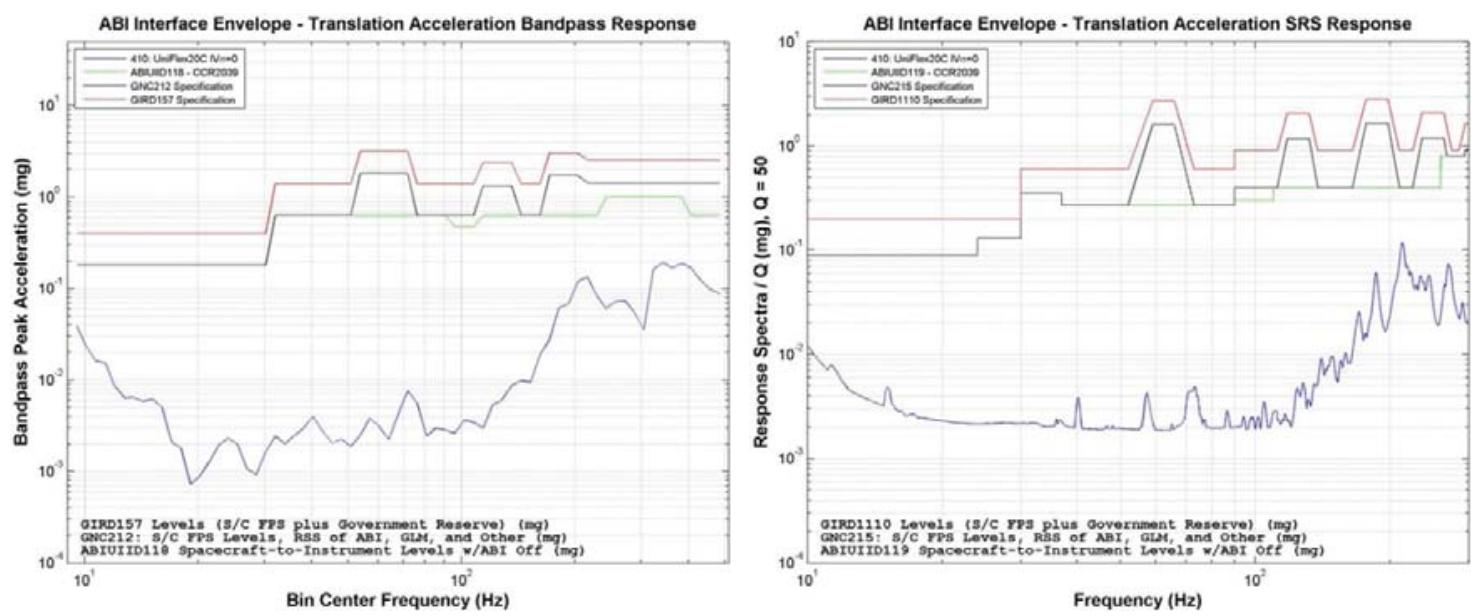

Fig. 16: ABI Mounting Feet Interface Accelerations with ABI Cryo-Coolers Disabled

When $\mathrm{ABI}$ is turned on and operating with a single cryo-cooler, the acceleration responses degrade dramatically. Fig. 17 shows envelope plots of the acceleration bandpass responses from 15 different simulations with single cryo-coolers running at different drive frequencies. The cryo-cooler drive frequency and its harmonics clearly show up as spikes in both the acceleration bandpass and the SRS responses. The simulated interface acceleration responses for ABI enabled show compliance with the GIRD157 requirements at all frequencies except around $310 \mathrm{~Hz}$ in the acceleration bandpass response. At this frequency, significant violations of both GNC212 and GIRD157 limits occur in the acceleration bandpass curve. Some smaller violations of the GNC212 and GNC215 limits occur at other frequencies for both the acceleration bandpass and the SRS requirements, respectively. However, other than the $310 \mathrm{~Hz}$ region of the acceleration bandpass response, the GIRD157 limit is met. The GIRD1110 SRS response limit is met at all frequencies.
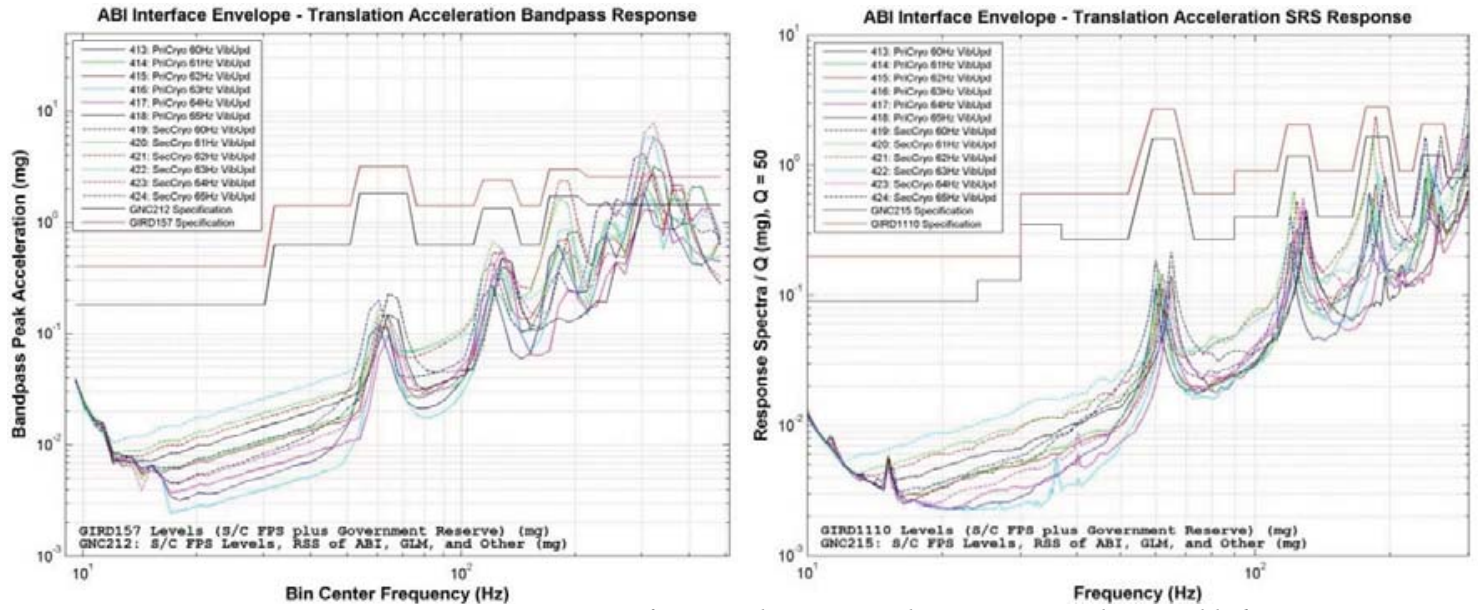

Fig. 17: ABI Mounting Feet Interface Accelerations with ABI Cryo-Coolers Enabled

The same analysis approach using the identical disturbance modeling was applied to assess the acceleration response of the GLM mounting feet with the ABI instrument operating. Fig. 18 shows the results. Here, only the GNC212 limit is violated at the $310 \mathrm{~Hz}$ frequency; the GIRD157 limit is not exceeded. In the SRS response, both the GNC215 limit and the GIRD1110 limit are met. 

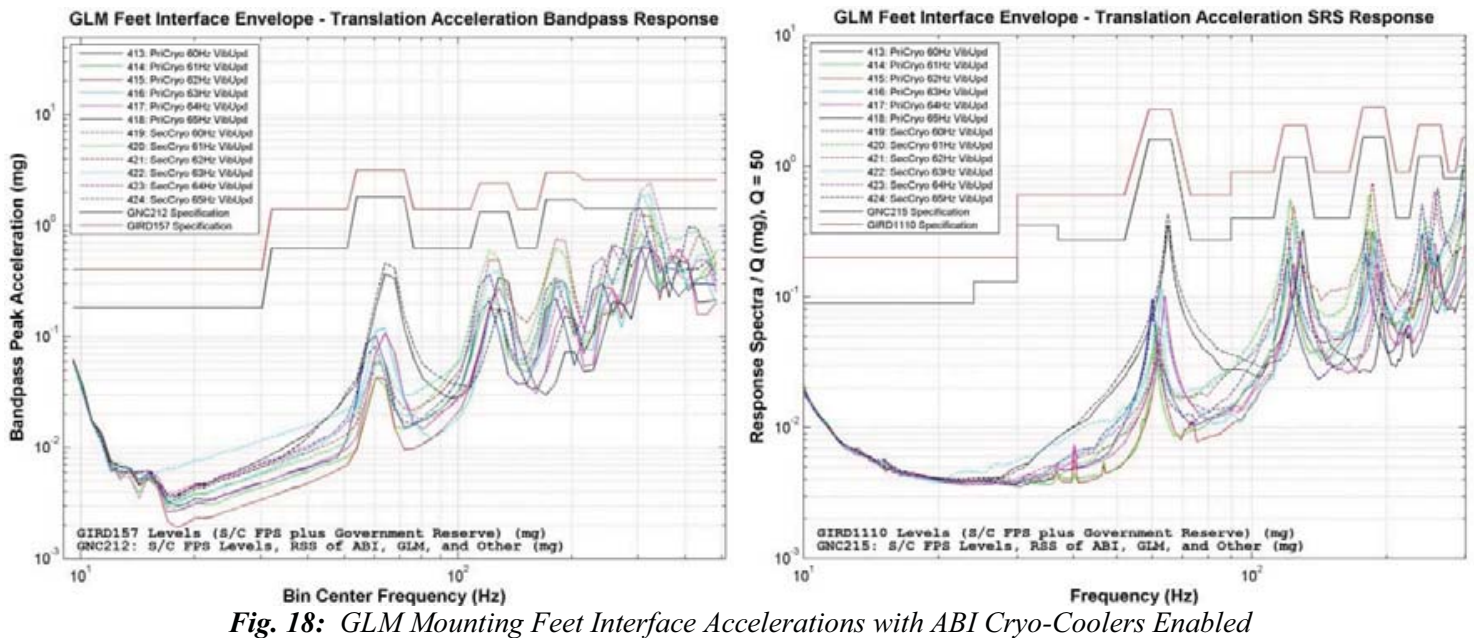

\section{EVALUATION OF GOES-R LINE-OF-SIGHT GUIDELINES}

In light of the possible exceedances in the interface acceleration requirements, the Flight Project developed LOS guidelines for both the ABI and GLM instruments. The LOS guidelines for both instruments are shown in Table 3. These guidelines represent a possible alternative specification for allowable jitter. Note that the GLM pointing stability guidelines indicate levels where GLM could sense false lightning events. Evaluation of the LOS performance is only possible with a high-fidelity, integrated structural model of the instruments and spacecraft designs, including optical degrees-of-freedom within the instruments. Enhanced structural models of the instruments were made available for this analysis, and incorporated into the high-fidelity jitter simulation.

Table 3: Earth-Observing Instrument LOS Jitter Guidelines

\begin{tabular}{|c|c|c|c|}
\hline Instrument & ABI On/Off & Axis & LOS Jitter (micro-rad) \\
\hline \multirow{3}{*}{ ABI } & \multirow{2}{*}{ ON } & NS (3-sigma) & 1.53 \\
\cline { 3 - 4 } & \multirow{2}{*}{ OFF } & EW (3-sigma) & 2.10 \\
\cline { 2 - 4 } & \multirow{2}{*}{ GLM (3-sigma) } & 1.46 \\
\hline \multirow{2}{*}{ On or Off } & EW (3-sigma) & 1.89 \\
\hline
\end{tabular}

The same disturbance models that were used previously in the evaluation of the interface acceleration requirements have also been used for the LOS simulations. The results of the LOS assessment are shown in Table 4. The ABI LOS is shown for both East-West and North-South directions, whereas the GLM LOS is shown as a total error. The assessment was performed for various cryo-cooler operating frequencies. As seen from Table 4, the GLM LOS guidelines are met with large margins for any cryo-cooler operating frequency. However, ABI LOS guidelines are not met for one of the six cryo-cooler frequencies shown. The North-South direction appears to be more sensitive than the East-West direction, although the LOS guidelines are also somewhat looser in the East-West direction.

The results imply a sensitivity of the ABI instrument LOS performance to the operating frequency of the cryocooler. Furthermore, the cryo-cooler frequencies are only spaced apart by $1 \mathrm{~Hz}$, so a small change in cryocooler frequency could result in significantly differently LOS performance on orbit. To further assess this and to validate the modeling, the GOES-R program will perform a full-up test of the ABI instrument dynamic response after installation on the spacecraft. This test, known as the Dynamic Interaction Test, will be performed in late 2014 or early 2015 . 
Table 4: ABI and GLM LOS Jitter Simulation Results

\begin{tabular}{|c|c|c|c|c|c|c|c|c|c|c|c|c|c|c|c|c|c|}
\hline \multirow{3}{*}{$\begin{array}{c}\text { ABI Cryocooler } \\
\text { State }\end{array}$} & \multirow{3}{*}{$\begin{array}{c}\text { Primary } \\
\text { Cryo Freq } \\
(\mathrm{Hz})\end{array}$} & \multirow{3}{*}{$\begin{array}{c}\text { Secondary } \\
\text { Cryo Freq } \\
(\mathrm{Hz})\end{array}$} & \multirow{3}{*}{$\begin{array}{c}\text { SA \& Mag } \\
\text { Boom } \\
\text { Thermal } \\
\text { Snap }\end{array}$} & \multirow{3}{*}{\begin{tabular}{|c|} 
RW \\
Isolation \\
Temp ("C)
\end{tabular}} & \multirow{3}{*}{$\begin{array}{l}\text { RW IV Dist. } \\
\text { Magnitude } \\
\text { (o) }\end{array}$} & \multicolumn{8}{|c|}{ ABI LOS Jitter (micro-radians) } & \multirow{2}{*}{\multicolumn{4}{|c|}{$\begin{array}{c}\text { GLM LOS Jitter (micro-radians) } \\
\text { Boresight }\end{array}$}} \\
\hline & & & & & & \multicolumn{4}{|c|}{ North-South } & \multicolumn{4}{|c|}{ East-West } & & & & \\
\hline & & & & & & 3-sigma & Margin & 3-sigma Limit & Peak & 3-sigma & Margin & 3-sigma Limit & Peak & 3-sigma & Peak & Margin & Peak Limit \\
\hline Off & $\cdot$ & - & On & 20 & 0.0 & 0.376 & $74 \%$ & 1.46 & 0.591 & 0.376 & $80 \%$ & 1.89 & 0.618 & 0.061 & 0.097 & $84 \%$ & \\
\hline VibeUpdate & 60 & - & On & 20 & 0.0 & 1.346 & $12 \%$ & & 2.231 & 1.312 & $38 \%$ & & 2.174 & 0.136 & 0.242 & $60 \%$ & \\
\hline VibeUpdate & 61 & - & On & 20 & 0.0 & 2.080 & $-36 \%$ & & 3.236 & 1.233 & $41 \%$ & & 2.078 & 0.134 & 0.243 & $60 \%$ & \\
\hline VibeUpdate & 62 & - & On & 20 & 0.0 & 1.426 & $7 \%$ & & 2.519 & 1.399 & $33 \%$ & & 2.083 & 0.129 & 0.223 & $63 \%$ & \\
\hline VibeUpdate & 63 & - & On & 20 & 0.0 & 1.469 & $4 \%$ & & 2.423 & 1.432 & $32 \%$ & & 2.287 & 0.129 & 0.235 & $61 \%$ & \\
\hline VibeUpdate & 64 & - & On & 20 & 0.0 & 1.275 & $17 \%$ & & 2.101 & 1.133 & $46 \%$ & & 1.921 & 0.128 & 0.237 & $61 \%$ & \\
\hline VibeUpdate & 65 & . & On & 20 & 0.0 & 1.060 & $31 \%$ & & 1.788 & 1.051 & $50 \%$ & & 1.908 & 0.150 & 0.272 & $55 \%$ & \\
\hline
\end{tabular}

\section{CONCLUSION}

The GOES-R spacecraft includes numerous features designed to optimize the INR performance, and to minimize jitter response of the earth-observing instruments. As shown in this paper, the attitude determination requirements are met with margin by utilizing star trackers and IMUs co-located with the instruments on a large optical bench. The high rate, low-latency SSIRU gyro data implementation is key to meeting the GOES-R unique IRE requirements. The Viceroy 4 GPSR development will provide a dramatic step forward in orbit determination performance at GEO. The GPS at GEO implementation for GOES-R represents an enabling technology for the imaging performance of the mission. The GOES-R program undertook the successful development of the LTR thruster, which allows torque balance between LTRs and RWAs during MA, NSSK, and EWSK maneuvers. The control law implementations, which include several unique feed-forward features and active vibration compensation, meet the pointing and pointing stability requirements even in the presence of MA, NSSK, EWSK, and instrument steering mirror disturbances. The designs provide the required "operatethrough" capability, resulting in unprecedented availability of Earth-observation data. Finally, the jitter interface acceleration requirements are generally met, except the acceleration bandpass requirements for $\mathrm{ABI}$ over a fairly narrow range around $310 \mathrm{~Hz}$. Closer examination of the instrument LOS performance shows that GLM guidelines are met, and ABI guidelines are met for most cryo-cooler operating frequencies. The program is pursuing a high-fidelity test program that will validate the jitter modeling approach, and will examine experimentally the jitter response of the instruments in detail. Successful completion of the test program will allow conservatism to be removed from the modeling, and will provide confidence that the on-orbit jitter response requirements will be met. As shown in this paper, the GN\&C performance for the GOES-R series of spacecraft supports the challenging mission objectives of the next generation GEO Earth-observation satellites.

\section{ACKNOWLEDGEMENTS}

This work was performed at Lockheed Martin Space Systems, under NASA contract NNG09HR00C, and at the National Aeronautics and Space Administration Goddard Space Flight Center. The authors gratefully acknowledge the many individuals who contributed in various ways through GOES-R program workshops and reviews, and helped developed a better understanding between instrument operations and spacecraft performance.

\section{REFERENCES}

[1] T. J. Schmit, Jun Li, Jinlong Li, W. F. Feltz, J. J. Gurka, M. D. Goldberg, K. J. Schrab, 2008: The GOES-R Advanced Baseline Imager and the Continuation of Current Sounder Products. J. Appl. Meteor. Climatol., 47, 2696-2711

[2] D. Freesland, D. Chu, A. Reth, A. Krimchansky, M. Donnelly, G. Comeyne, "Advancing the Next Generation GOES-R Operational Availability,” Proceedings of the 2005 AAS Guidance and Control Conference, Breckenridge, CO, Feb 2005

[3] GOES-R Functional and Performance Specification (F\&PS), 417-R-PSPEC-0014, Version 2.13, November $17,2010$.

[4] A. A. Kamel, "GOES Image Navigation and Registration System," In GOES-8 and Beyond, Edward R. Washwell, Editor, Proc. SPIE 2812, (1996), pp 766-776.

[5] D. Igli, V. Virgilio and K Grounder, "Image Navigation and Registration for GOES-R Advanced Baseline Imager," Proceedings of the 2009 AAS G\&C Conference, Breckenridge, CO, Feb 2009.

[6] D. Igli, "GOES-R Advanced Baseline Imager Precise Pointing Control and Image Collection", Proceedings of the 2013 AAS Guidance and Control Conference, Breckenridge, CO, Feb 2013. 
[7] D. Freesland, A. Reth, A. Krimchansky, M. Donnelly, Tim Walsh, "Improving Attitude Stability Performance on GOES-R Using PIFT," Proceedings of the 2007 AAS Guidance and Control Conference, Breckenridge, CO, Feb 2007.

[8] L. P. Davis, D. R. Carter, T. T. Hyde, "Second-Generation Hybrid D-Strut," Proc. SPIE 2445, Smart Structures and Materials 1995: Passive Damping, 161, May 1995.

[9] C. D. Johnson, J. M. Howat, P. S. Wilke, "Vibration Isolation System and Method," U.S. Patent 20140084527A1, Moog, Inc., Publication Date Mar 27, 2014.

[10] N. E. Goodzeit, H. J. Weigl, "Active Vibration Damping (AVD) System for Precision Pointing Spacecraft," U.S. Patent WO2008136881A1, Lockheed Martin, Publication Date Nov 13, 2008.

[11] J. Chapel, E. Schmitz, W. Sidney, M. Johnson, P. Good, J. Wynn, T. Bayer, "Attitude Control Performance for MRO Aerobraking and the Initial Science Phase," Proceedings of the 2007 AAS Guidance and Control Conference, Breckenridge, CO, Feb 2007.

[12] E. J. Lefferts, F. L. Markley, and M. D. Shuster, "Kalman Filtering for Spacecraft Attitude Estimation," Journal of Guidance, Sep-Oct, 1982, Vol 5, No 5, p. 417.

[13] Shuster, M. D. (1990). Kalman filtering of spacecraft attitude and the QUEST model. Journal of the Astronautical Sciences, 38, 377-393.

[14] D. Piot, L. Oddos-Marcel, B. Gelin, A. Thieuw, P. Genty, P. Martinez, S. Airey, "HYDRA Star Tracker Onboard SPOT-6," Proceedings of the 2013 AAS Guidance and Control Conference, Breckenridge, CO, Feb 20013.

[15] S. Winkler, C. Voboril, R. Hart, M. King, "GOES-R Use of GPS at GEO (Viceroy-4)," Proceedings of the 2013 AAS Guidance and Control Conference, Breckenridge, CO, Feb 2013.

[16] L. Barker, "GPS Beyond LEO: Signal Environment System Design Considerations". Proceedings of the 2008 AAS Guidance and Control Conference, Breckenridge, CO, Feb 2008.

[17] K. Larson, D. Gaylor, S. Winkler, "Worst-Case GPS Constellation for Testing Navigation at Geosynchronous Orbit for GOES-R," Proceedings of the 2013 AAS Guidance and Control Conference, Breckenridge, CO, Feb 2013. 